\title{
On automorphism groups of graph truncations
}

\author{
Brian Alspach \\ School of Mathematical and Physical Sciences \\ University of Newcastle \\ Callaghan, NSW 2308 \\ Australia \\ Edward Dobson \\ Departmentment of Mathematics and Statisitics \\ Mississippi State University \\ Mississippi State, MS 39762 USA \\ and \\ UP IAM \\ University of Primorska \\ Muzejeska trg 2, 6000 Koper, Slovenia
}

Received 11 May 2014, accepted 13 November 2014, published online 17 December 2014

\begin{abstract}
It is well known that the Petersen graph, the Coxeter graph, as well as the graphs obtained from these two graphs by replacing each vertex with a triangle, are trivalent vertextransitive graphs without Hamilton cycles, and are indeed the only known connected vertextransitive graphs of valency at least two without Hamilton cycles. It is known by many that the replacement of a vertex with a triangle in a trivalent vertex-transitive graph results in a vertex-transitive graph if and only if the original graph is also arc-transitive. In this paper, we generalize this notion to $t$-regular graphs $\Gamma$ and replace each vertex with a complete graph $K_{t}$ on $t$ vertices. We determine necessary and sufficient conditions for $\mathscr{T}(\Gamma)$ to be hamiltonian, show $\operatorname{Aut}(\mathscr{T}(\Gamma)) \cong \operatorname{Aut}(\Gamma)$, as well as show that if $\Gamma$ is vertex-transitive, then $\mathscr{T}(\Gamma)$ is vertex-transitive if and only if $\Gamma$ is arc-transitive. Finally, in the case where $t$ is prime we determine necessary and sufficient conditions for $\mathscr{T}(\Gamma)$ to be isomorphic to a Cayley graph as well as an additional necessary and sufficient condition for $\mathscr{T}(\Gamma)$ to be vertex-transitive.
\end{abstract}

Keywords: Truncation, automorphism group, Cayley graph, Hamiltonian.

Math. Subj. Class.: 05C25

E-mail addresses: brian.alspach@newcastle.edu.au (Brian Alspach),dobson@math.msstate.edu (Edward Dobson)

(a) (i) This work is licensed under http://creativecommons.org/licenses/by/3.0/ 


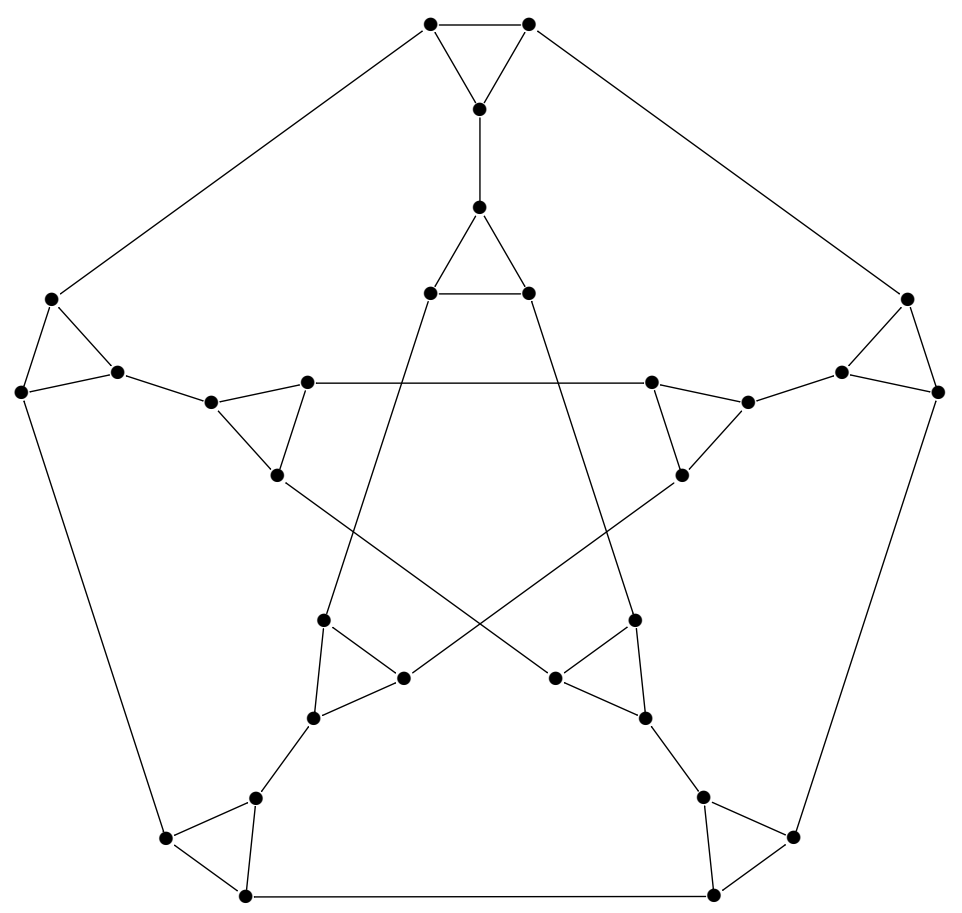

Figure 1: The truncation of the Petersen graph

\section{Introduction}

In 1969 Lovász posed the problem below (this statement is written exactly as Lovász wrote it).

Problem 1.1 (Lovász, 1969). Let us construct a finite, connected, undirected graph which is symmetric and has no simple path containing all elements. A graph is called symmetric, if for any two vertices $x, y$ it has an automorphism mapping $x$ into $y$.

Usually this problem is stated as the conjecture that every vertex-transitive graph contains a Hamilton path (here "vertex-transitive" and "symmetric" are synonyms). Typically though it is usually the case that this conjecture is verified by showing that a a particular vertextransitive graph contains a Hamilton cycle. Much work has been done in attempting to verify this conjecture - see [5] for some recent information regarding progress on this conjecture. The Petersen graph is vertex-transitive but does not contain a Hamilton cycle (see for example [3, Theorem 1.5.1]), while Tutte [9] first showed that the Coxeter graph is not hamiltonian, with an additional proof by Biggs [1]. The graphs obtained from the Petersen graph and the Coxeter graph by replacing each vertex with a triangle — called the truncation - are also vertex-transitive graphs that do not contain a Hamilton cycle. These four graphs are the only known connected vertex-transitive graphs, other than $K_{2}$, that do not have a Hamilton cycle. The truncation of the Petersen graph is shown in Figure 1.

It turns out that the truncation of the truncation of the Petersen and Coxeter graphs are not vertex-transitive. It is known by many that the truncation of a trivalent graph $\Gamma$ 
is vertex-transitive if and only if $\operatorname{Aut}(\Gamma)$ is also transitive on the edges of $\Gamma$, or edgetransitive, although neither of the previously stated facts are proven in the literature. We will generalize the notion of truncation to vertex-transitive graphs of valencies other than 3 . Note that as a triangle can be viewed as either a cycle of length 3 or a complete graph $K_{3}$, there are two natural generalizations of the idea of truncation. Namely, one can "replace" each vertex with a cycle or with a complete graph, or even with an arbitrary graph. These have been studied for example in [2, 6, 11] We note that in [11], the graph obtained by replacing each vertex with a complete graph is called a clique-inserted graph, and that replacing each vertex with a cycle is motivated by map truncation. For a vertex $v \in V(\Gamma)$, we denote the valency of $v$ in $\Gamma$ by $\operatorname{val}(v)$.

Definition 1.2. Let $\Gamma$ be a graph. The truncation $\mathscr{T}(\Gamma)$ of $\Gamma$ is obtained from $\Gamma$ by replacing each vertex $v$ of $\Gamma$ with a clique on $\operatorname{val}(v)$ vertices, denoted $T_{v}$, and whenever $u v \in E(\Gamma)$, then one vertex of $T_{v}$ is adjacent to one vertex of $T_{u}$ and no vertex of $T_{v}$ is adjacent to more than one vertex outside of $T_{v}$.

Note that if $u v \in E(\Gamma)$, we do not specify which vertex of $T_{u}$ is adjacent to $T_{v}$. Obviously, different choices of such vertices will result in different graphs, but all such choices result in isomorphic graphs as each $T_{u}$ and $T_{v}$ is a complete graph and no vertex of $T_{v}$ is adjacent to more than one vertex outside of $T_{v}$. Also, as each vertex in $T_{v}$ is adjacent to $\operatorname{val}(v)-1$ vertices inside $T_{v}$ and exactly one vertex outside $T_{v}$, a vertex $u \in T_{v}$ has $\operatorname{valency} \operatorname{val}(v)$ in the truncation $\mathscr{T}(\Gamma)$. In particular, the truncation of a $t$-regular graph is still $t$-regular.

We should point out that there is an equivalent definition of graph truncation introduced in [7]. For a graph $\Gamma$, let $S(\Gamma)$ be the graph obtained from $\Gamma$ by subdividing each edge via the insertion of a single vertex, and $L(\Gamma)$ be the line graph of $\Gamma$. Then $\mathscr{T}(\Gamma)=L(S(\Gamma))$.

In this paper we show $\mathscr{T}(\Gamma)$ contains a Hamilton cycle if and only if $\Gamma$ has a spanning eulerian subgraph (Theorem 2.1). We then show that for a graph $\Gamma$ with minimal valency at least 3 the automorphism group of its truncation is isomorphic to $\operatorname{Aut}(\Gamma)$ (Theorem 3.6), and subsequently that a connected vertex-transitive graph with minimal valency at least 3 has a vertex-transitive truncation if and only if it is arc-transitive (Theorem 3.7), or transitive on the set of directed edges or arcs of $\Gamma$. We remark that this is consistent with the statements earlier that a trivalent vertex-transitive graph has vertex-transitive truncation if and only if it is edge-transitive as a vertex- and edge-transitive graph of odd valency is necessarily arc-transitive [10, 7.53]. Finally, for a vertex-transitive graph $\Gamma$ of prime valency, we also determine necessary and sufficient conditions for $\mathscr{T}(\Gamma)$ be a Cayley graph provided that $\mathscr{T}(\Gamma)$ is vertex-transitive (Theorem 3.8), as well as provide an alternative characterization of when $\mathscr{T}(\Gamma)$ is vertex-transitive (Theorem 3.10). We begin with necessary and sufficient conditions for $\mathscr{T}(\Gamma)$ to be Hamiltonian.

\section{Hamiltonicity of Graph Truncations}

Theorem 2.1. If $\Gamma$ is a graph, then $\mathscr{T}(\Gamma)$ contains a Hamilton cycle if and only if $\Gamma$ contains a connected spanning eulerian subgraph.

Proof. First suppose that $\Gamma$ has a connected spanning eulerian subgraph $\Delta$, and let $v_{0} v_{1} \ldots$ $v_{r} v_{0}$ be an Euler tour of $\Delta$, where we traverse the tour so that the edge $v_{i} v_{i+1}$ is traversed from $v_{i}$ to $v_{i+1}$. Given that the edge $v_{i} v_{i+1}$ is traversed from $v_{i}$ to $v_{i+1}$, let $u_{i, 0}$ and $u_{i+1,1}$ denote the vertices of $T_{v_{i}}$ and $T_{v_{i+1}}$, respectively, that are adjacent. For each $x \in V(\Gamma)$, we 
let $x_{m}$ be the largest nonnegative integer for which $v_{x_{m}}=x$, set $Y_{x}=\left\{i<x_{m}: v_{i}=x\right\}$, and $Z_{x}=\left\{u_{i, 0}, u_{i, 1}: i \in Y_{x}\right\}$. For each $1 \leq i \leq r$ construct a path $P_{i}$ as follows: Setting $x=v_{i}$, we let $P_{i}=u_{i, 0}$ if $i<x_{m}$, while if $i=x_{m}$, we let $Q_{i}$ be a Hamilton path from $u_{i, 1}$ to $u_{i, 0}$ in $\mathscr{T}(\Gamma)\left[T_{x}-Z_{x}\right]$, the subgraph of $\mathscr{T}(\Gamma)$ induced by $T_{x}-Z_{x}$. Let $P_{i}$ be the path obtained from $Q_{i}$ by removing the initial vertex $u_{i, 1}$ of $Q_{i}$. We observe that $Q_{i}$ and consequently $P_{i}$ certainly exist as $\mathscr{T}(\Gamma)\left[T_{x}-Z_{x}\right]$ is a clique. Then

$$
u_{0,0} u_{1,1} P_{1} u_{1,0} u_{2,1} P_{2} u_{2,0} \ldots u_{r, 1} P_{r} u_{r, 0} u_{0,1} P_{0} u_{0,0}
$$

is a Hamilton cycle in $\mathscr{T}(\Gamma)$. Intuitively, we travel along the Euler tour until the last time we visit a $T_{v}$, at which point we traverse all the previously unvisited vertices of $T_{v}$.

Conversely, suppose that $H=v_{0} v_{1} \ldots v_{n}$ is a Hamilton cycle in $\mathscr{T}(\Gamma)$ (so $v_{n}=v_{0}$ ). For each $0 \leq i \leq n$, let $v_{i} \in T_{x_{i}}, x_{i} \in V(\Gamma)$. Let $E^{\prime}=\left\{x_{i} x_{i+1}: T_{x_{i}} \neq T_{x_{i+1}}\right\}$, so that $E^{\prime}$ is simply the set of edges of $H$ that connect different inserted cliques. Then the edges of $E^{\prime}$ form a spanning connected subgraph of $\Gamma$ as $H$ is a Hamilton cycle in $\mathscr{T}(\Gamma)$. Additionally, with the exception of $T_{x_{0}}$ and $T_{x_{n}}=T_{x_{0}}$, each time one traverses $H$ and enters a $T_{x}$, one must exit that $T_{x}$. We conclude that every vertex of the graph formed by the edges of $E^{\prime}$ has even valency and so this graph is eulerian.

In the case of trivalent graphs, as the only spanning eulerian subgraph of a trivalent graph is necessarily a Hamilton cycle, we have the following result.

Corollary 2.2. The truncation of a trivalent graph $\Gamma$ is hamiltonian if and only if $\Gamma$ is hamiltonian.

As the Petersen graph and the Coxeter graph are both trivalent graphs that are not hamiltonian, the following result is evident.

Corollary 2.3. The truncations of the Petersen graph and the Coxeter graph are not hamiltonian.

\section{Vertex-transitive Graph Truncations}

While the truncation of any vertex-transitive trivalent graph that is not hamiltonian will give a trivalent graph that is not hamiltonian, the truncation of such a graph need not be vertex-transitive. Indeed, the truncations of the truncations of the Petersen and Coexeter graphs are not hamiltonian, but it turns out that they are not vertex-transitive. We now investigate when the truncation of a vertex-transitive graph is vertex-transitive. We begin by studying the relationship between $\operatorname{Aut}(\Gamma)$ and $\operatorname{Aut}(\mathscr{T}(\Gamma))$ for any graph $\Gamma$.

Definition 3.1. Let $\Gamma$ be a graph. We call the set $\mathcal{T}=\left\{T_{v}: v \in V(\Gamma)\right\}$ the fundamental vertex partition of $V(\mathscr{T}(\Gamma)$. There is also a fundamental edge partition of $E(\mathscr{T}(\Gamma))$ with two cells, where one cell consists of those edges within a $T_{v}, v \in V(\Gamma)$ (the clique edges), and the other cell consisting of those edges between two inserted cliques (the original edges).

Lemma 3.2. Let $\Gamma$ be a graph with each vertex of valency at least 3 . Then the fundamental vertex and edge partitions of $\mathscr{T}(\Gamma)$ are invariant under $\operatorname{Aut}(\mathscr{T}(\Gamma))$. 
Proof. We need only show that the fundamental vertex partition of $\mathscr{T}(\Gamma)$ is invariant under $\operatorname{Aut}(\mathscr{T}(\Gamma))$, as this implies that the fundamental edge partition is invariant under $\operatorname{Aut}(\mathscr{T}(\Gamma))$. An edge $x y$ with $x \in V\left(T_{v}\right)$ and $y \in V\left(T_{u}\right), u \neq v$, cannot belong to a triangle because $y$ is the only neighbor of $x$ not in $V\left(T_{v}\right)$ and $x$ is the only neighbor of $y$ not in $V\left(T_{u}\right)$. On the other hand, every edge $x y$ with $x, y$ in the same $V\left(T_{v}\right)$ belongs to a triangle because $T_{v}$ is a clique and $t \geq 3$. This then implies that $\operatorname{Aut}(\mathscr{T}(\Gamma))$ permutes the sets in the partition $\mathcal{T}=\left\{V\left(T_{v}\right): v \in V(\Gamma)\right\}$.

We now introduce standard permutation group theoretic terms related to the fundamental vertex partition.

Definition 3.3. Let $G \leq S_{n}$ be transitive and act on $\mathbb{Z}_{n}$. Let $B \subseteq \mathbb{Z}_{n}$. If $g(B)=B$ or $g(B) \cap B=\emptyset$ for every $g \in G$, then $B$ is a block of $G$. If $B$ is a block, then $g(B)$ is also a block for all $g \in G$, and $\{g(B): g \in G\}$ is a $G$-invariant partition. Of course, singleton sets and $\mathbb{Z}_{n}$ are blocks for every transitive group $G \leq S_{n}$, and the corresponding $G$-invariant partitions are trivial. If $G$ has a nontrivial $G$-invariant partition, then $G$ is imprimitive, and is primitive otherwise. Finally, for a $G$-invariant partition $\mathcal{B}$, we denote by $\operatorname{fix}_{G}(\mathcal{B})$ the subgroup of $G$ which fixes each block of $\mathcal{B}$ setwise. That is, $\operatorname{fix}_{G}(\mathcal{B})=$ $\{g \in G: g(B)=B$ for all $B \in \mathcal{B}\}$.

We remark that if $\operatorname{Aut}(\mathscr{T}(\Gamma))$ is transitive, then the fundamental vertex partition is an $\operatorname{Aut}(\mathscr{T}(\Gamma))$-invariant partition.

Now observe that if $\Gamma$ is a cycle of length $n$, then $\mathscr{T}(\Gamma)$ is a cycle of length $2 n$. Hence $\operatorname{Aut}(\mathscr{T}(\Gamma))=D_{2 n}$, the dihedral group of order $4 n$. Henceforth, we will assume that every vertex has valency at least 3 , in which case $\mathscr{T}(\Gamma)$ always contains a triangle.

Theorem 3.4. Let $\Gamma$ be a graph where each vertex of $\Gamma$ has valency at least 3 . Then $\operatorname{Aut}(\mathscr{T}(\Gamma))$ acts faithfully on the fundamental vertex partition $\mathcal{T}$ and is isomorphic to a subgroup of $\operatorname{Aut}(\Gamma)$.

Proof. That $\operatorname{Aut}(\Gamma)$ acts on $\mathcal{T}$ was established in Lemma 3.2. Let $K$ be the kernel of the action of $\operatorname{Aut}(\mathscr{T}(\Gamma))$ on $\mathcal{T}$ (if $\operatorname{Aut}(\mathscr{T}(\Gamma))$ is transitive, then $K=\operatorname{fix}_{\operatorname{Aut}(\mathscr{T}(\Gamma))}(\mathcal{T})$ ). We claim that $K=1$. Indeed, if $K \neq 1$ with $1 \neq \gamma \in K$, then let $T_{v} \in \mathcal{T}$ such that $\left.K\right|_{T_{v}} \neq 1$. Then there exist distinct $x, y \in T_{v}$ such that $\gamma(x)=y$. Now, $x$ is adjacent to some vertex $z \in T_{u}, u \neq v$, and so $\gamma(x) \gamma(z)$ is also an edge from $T_{v}$ to $T_{u}$. However, there is only one edge from a vertex of $T_{v}$ to a vertex of $T_{u}$ in $\mathscr{T}(\Gamma)$, a contradiction. Thus $K=1$.

To finish the result, we need only show that if $\gamma \in \operatorname{Aut}(\mathscr{T}(\Gamma))$, then $\bar{\gamma} \in \operatorname{Aut}(\Gamma)$, where $\bar{\gamma}$ is the induced action of $\gamma$ on $\mathcal{T}$. So suppose that $u v \in E(\Gamma)$. Then some vertex of $T_{u}$ is adjacent to some vertex of $T_{v}$, and as $\gamma \in \operatorname{Aut}(\mathscr{T}(\Gamma))$, some vertex of $\gamma\left(T_{u}\right)=T_{\bar{\gamma}(u)}$ is adjacent to some vertex of $\gamma\left(T_{v}\right)=T_{\bar{\gamma}(v)}$. But this occurs if and only if $\bar{\gamma}(u) \bar{\gamma}(v) \in$ $E(\Gamma)$.

Corollary 3.5. The truncations of the truncations of the Petersen and Coxeter graphs are not vertex-transitive.

Proof. Let $\Gamma$ be the Petersen or Coexeter graph. If $\mathscr{T}(\mathscr{T}(\Gamma))$ is vertex-transitive, then 9 divides $|\operatorname{Aut}(\mathscr{T}(\mathscr{T}(\Gamma)))|$ as $|V(\mathscr{T}(\mathscr{T}(\Gamma)))|=9|V(\Gamma)|$ and the size of an orbit or a group divides the order of the group. By Theorem 3.4 applied twice, we see that 9 divides $|\operatorname{Aut}(\Gamma)|$. However, the automorphism group of the Petersen graph has order 120 as it is isomorphic to $S_{5}$ (see for example [3, Theorem 1.4.6]) while the automorphism group of 
the Coexeter graph has order 336 as it is $\operatorname{PGL}(2,7)$ (see for example [1]), neither of which are divisible by 9 .

Theorem 3.6. If $\Gamma$ is a graph with each vertex of valency at least 3 , then $\operatorname{Aut}(\mathscr{T}(\Gamma)) \cong$ $\operatorname{Aut}(\Gamma)$.

Proof. In view of Theorem 3.4, it suffices to show that each element of Aut $(\Gamma)$ induces an element of $\operatorname{Aut}(\mathscr{T}(\Gamma))$, and that different elements of $\operatorname{Aut}(\Gamma)$ induce different elements of $\operatorname{Aut}(\mathscr{T}(\Gamma))$.

Let $\gamma \in \operatorname{Aut}(\Gamma)$. Let $x \in V(\mathscr{T}(\Gamma))$, and $v \in V(\Gamma)$ with $x \in T_{v}$. Then there exists a unique $y \in V(\mathscr{T})$ not contained in $T_{v}$ with $x y \in E(\mathscr{T}(\Gamma))$. Let $u \in V(\Gamma)$ such that $y \in T_{u}$. Then $\gamma(u) \gamma(v) \in E(\Gamma)$, and so there exists vertices $x^{\prime} \in T_{\gamma(v)}$ and $y^{\prime} \in T_{\gamma(u)}$ such that $x^{\prime} y^{\prime} \in E(\mathscr{T}(\Gamma))$. Define $\bar{\gamma}: V(\mathscr{T}(\Gamma)) \mapsto(\mathscr{T}(\Gamma))$ by $\bar{\gamma}(x)=x^{\prime}$. Note that as the original edges of $\mathscr{T}(\Gamma)$ form a perfect matching, $\bar{\gamma}$ is a well-defined bijection. Additionally, by definition, $\bar{\gamma}$ maps the original edges of $\mathscr{T}(\Gamma)$ to the original edges of $\mathscr{T}(\Gamma)$. As $\bar{\gamma}$ also map the fundamental vertex partition of $\mathscr{T}(\Gamma)$ to itself, it maps the clique edges of $\mathscr{T}(\Gamma)$ to the clique edges of $\mathscr{T}(\Gamma)$. Thus $\bar{\gamma} \in \operatorname{Aut}(\mathscr{T}(\Gamma)$. Finally, as the induced action of $\bar{\gamma}$ on $\mathcal{T}$ is $\gamma$ and $\operatorname{Aut}(\mathscr{T}(\Gamma)$ is faithful on $\mathcal{T}$ by Lemma 3.2, different elements of $\operatorname{Aut}(\Gamma)$ induce different elements of $\operatorname{Aut}(\mathscr{T}(\Gamma)$.

For a transitive group $G$ acting on $\mathbb{Z}_{n}$, we denote the stabilizer in $G$ of $v$ by $\operatorname{Stab}_{G}(v)$. Then $\operatorname{Stab}_{G}(v)=\{g \in G: g(v)=v\}$. Concerning the statement of the following result, a vertex- and edge-transitive graph of odd valency is necessarily arc-transitive [10, 7.53].

Theorem 3.7. If $\Gamma$ is a connected vertex-transitive graph with each vertex of valency $t \geq 3$, then $\mathscr{T}(\Gamma)$ is vertex-transitive if and only if $\Gamma$ is arc-transitive. Additionally, $\mathscr{T}(\mathscr{T}(\Gamma))$ is not vertex-transitive.

Proof. Before proceeding, some general observations about $\mathscr{T}(\Gamma)$ are in order. As $\mathscr{T}(\Gamma)$ is regular of valency $t, \mathscr{T}(\Gamma)\left[T_{v}\right]$ is regular of valency $t-1$. As $\left|V\left(T_{v}\right)\right|=t$, we see that there are exactly $t$ edges with one end in $T_{v}$ and the other end not in $T_{v}$, and of course each vertex of $T_{v}$ is incident with exactly one such edge. Additionally, between some vertex of $T_{v}$ and some vertex of $T_{u}$ there is either exactly one edge if $u v \in E(\Gamma)$ or no edges if $u v \notin E(\Gamma)$. Thus, each edge with one endpoint in $T_{v}$ and the other endpoint outside of $T_{v}$ uniquely determines a vertex in $T_{v}$ and uniquely determines a $T_{u}$ in which the other endpoint of the edge is a vertex. Conversely, each vertex $x$ of $T_{v}$ uniquely determines an edge with $x$ as an endpoint and one endpoint not in $T_{v}$.

Suppose that $\Gamma$ is arc-transitive, with $v \in V(\Gamma)$. Then $\operatorname{Stab}_{\operatorname{Aut}(\Gamma)}(v)$ is transitive on the neighbors $N_{\Gamma}(v)$ of $v$. Set $N_{\Gamma}(v)=\left\{u_{1}, \ldots, u_{t}\right\}$ and let $\gamma_{i, j} \in \operatorname{Stab}_{\text {Aut }(\Gamma)}(v)$ such that $\gamma_{i}\left(u_{i}\right)=u_{j}$. As $\operatorname{Aut}(\Gamma) \cong \operatorname{Aut}(\mathscr{T}(\Gamma))$ by Theorem 3.6 and $\operatorname{Aut}(\mathscr{T}(\Gamma))$ acts faithfully on $\mathcal{T}=\left\{T_{v}: v \in V(\Gamma)\right\}$ by Theorem 3.4, there exists a unique $\hat{\gamma}_{i, j} \in \operatorname{Aut}(\mathscr{T}(\Gamma))$ such that the action of $\hat{\gamma}_{i, j}$ on $\mathcal{T}$ is $\gamma_{i, j}$. As the action of $\operatorname{Aut}(\mathscr{T}(\Gamma))$ on $\mathcal{T}$ is $\operatorname{Aut}(\Gamma)$ which is transitive, in order to show that $\operatorname{Aut}(\mathscr{T}(\Gamma))$ is transitive it suffices to show that $\{\delta \in$ $\left.\operatorname{Aut}(\mathscr{T}(\Gamma)): \delta\left(T_{v}\right)=T_{v}\right\}$ is transitive on $T_{v}$. Let $x, y \in T_{v}$. Then there exist $1 \leq i, j \leq t$ such that $x v_{u_{i}}, y v_{u_{j}} \in E(\mathscr{T}(\Gamma))$, where $v_{u_{i}} \in T_{u_{i}}$ and $v_{u_{j}} \in T_{u_{j}}$. Then $\hat{\gamma}_{i, j}\left(T_{u_{i}}\right)=T_{u_{j}}$ and $\hat{\gamma}_{i, j}\left(T_{v}\right)=T_{v}$. As each vertex of $T_{v}$ is incident with exactly one edge whose other endpoint is not in $T_{v}$ and $\hat{\gamma}_{i, j} \in \operatorname{Aut}(\mathscr{T}(\Gamma))$, we have that $\hat{\gamma}_{i, j}\left(x v_{u_{i}}\right)$ is the unique edge of $\mathscr{T}(\Gamma)$ with one endpoint in $T_{v}$ and the other in $T_{u_{j}}$. That is, $\hat{\gamma}_{i, j}\left(x v_{u_{i}}\right)=y v_{u_{j}}$. As 
$\hat{\gamma}_{i, j}\left(T_{v}\right)=T_{v}$, we conclude that $\hat{\gamma}_{i, j}(x)=y$. Thus $\left\{\delta \in \operatorname{Aut}(\mathscr{T}(\Gamma)): \delta\left(T_{v}\right)=T_{v}\right\}$ is transitive on $T_{v}$ and the result follows.

Conversely, suppose that $\mathscr{T}(\Gamma)$ is vertex-transitive. It suffices to show that the stabilizer in $\operatorname{Aut}(\Gamma)$ of $v \in V(\Gamma)$ is transitive on its neighbors. Observe that $\mathcal{T}=\left\{T_{v}: v \in V(\Gamma)\right\}$ is an $\operatorname{Aut}(\mathscr{T}(\Gamma))$-invariant partition and $H_{v}=\left\{h \in \operatorname{Aut}(\mathscr{T}(\Gamma)): h\left(T_{v}\right)=T_{v}\right\}, v \in V(\Gamma)$, is transitive on $T_{v}$. Hence, if $x, y \in V\left(T_{v}\right)$, then there exists $\gamma_{x, y} \in \operatorname{Aut}(\mathscr{T}(\Gamma))$ such that $\gamma_{x, y}(x)=y$. For each vertex $x$ of $T_{v}$, we denote the uniquely determined edge with $x$ as an endpoint and with the other endpoint not in $T_{v}$ by $e_{x}=x z_{x}$. We let $v_{x} \in V(\Gamma)$ be such that $z_{x} \in V\left(T_{v_{x}}\right)$, and observe that if $x, y \in V\left(T_{v}\right)$ with $x \neq y$, then $T_{v_{x}} \neq T_{v_{y}}$. Each edge $x z_{x}$ with $x \in V\left(T_{v}\right)$ then induces an edge $v v_{x} \in E(\Gamma)$, and such edges are pairwise distinct. More specifically, there are exactly $t$ edges $v v_{x} \in E(\Gamma)$ induced by edges of the form $x z_{x}$ with $x \in V\left(T_{v}\right)$. This then implies that the neighbors in $\Gamma$ of $v$ are $\left\{v_{x}: x \in V\left(T_{v}\right)\right\}$. Finally, observe that $\gamma_{x, y}\left(x z_{x}\right)$ is an edge with one endpoint $y \in V\left(T_{v}\right)$ and $\gamma_{x, y}\left(z_{x}\right) \notin V\left(T_{v}\right)$. Denoting by $\bar{\gamma}_{x, y}$ the automorphism of $V(\Gamma)$ induced by the action of $\gamma_{x, y}$ on $\mathcal{T}$ (with each $T_{a}$ identified with the vertex $a$ ), we see that $\bar{\gamma}_{x, y}\left(v v_{x}\right)=v v_{y}$, and the stabilizer of $v \in V(\Gamma)$ in $\operatorname{Aut}(\Gamma)$ is transitive on the neighbors of $v$ and so $\Gamma$ is arc-transitive.

It now only remains to show that $\mathscr{T}(\mathscr{T}(\Gamma))$ is not vertex-transitive. In view of our earlier arguments, it suffices to show that if $\Gamma$ is edge-transitive, then $\mathscr{T}(\Gamma)$ is not edgetransitive. If $\Gamma$ is edge-transitive, then $\operatorname{Aut}(\mathscr{T}(\Gamma))$ is transitive, and $\operatorname{Aut}(\mathscr{T}(\Gamma))$ admits $\mathcal{T}$ as an $\operatorname{Aut}(\Gamma)$-invariant partition. But $\mathscr{T}(\Gamma)$ contains edges with both endpoints in $T_{v}$ and edges with one endpoint in $T_{v}$ and one endpoint outside of $T_{v}$. As $\mathcal{T}$ is an $\operatorname{Aut}(\Gamma)-$ invariant partition, no automorphism will map an edge of the former type to an edge of the latter type.

We now restrict our attention to graphs with prime valency. We remark that in the following result, the restriction to prime valency is only used to establish sufficiency.

Theorem 3.8. If $\Gamma$ is a connected arc-transitive graph of prime valency $t \geq 3$ and order $n$, then $\mathscr{T}(\Gamma)$ is isomorphic to a Cayley graph if and only if $\operatorname{Aut}(\Gamma)$ contains a transitive group of order $n t$.

Proof. As a vertex-transitive graph is isomorphic to a Cayley graph if and only if its automorphism group contains a regular subgroup [8], $\mathscr{T}(\Gamma)$ being a Cayley graph implies that $\operatorname{Aut}(\mathscr{T}(\Gamma))$ contains a transitive group $R$ of order $n t$ which is a isomorphic to a transitive subgroup of $\operatorname{Aut}(\Gamma)$ of order $n t$ by Theorem 3.4.

Conversely suppose there exists $R \leq \operatorname{Aut}(\Gamma)$ such that $R$ is transitive on $V(\Gamma)$ and has order $n t$. It suffices to show that for fixed $v \in V(\Gamma)$, the subgroup $H$ of $R$ fixing the set $V\left(T_{v}\right)$ is transitive on $V\left(T_{v}\right)$, as then $R$ is transitive and as $|R|=|V(\mathscr{T}(\Gamma))|$, we have that $R$ is regular.

Now, $\mathcal{T}$ is an $\operatorname{Aut}(\mathscr{T}(\Gamma))$-invariant partition, and the action of $R$ on $\mathcal{T}$, which we denote by $R / \mathcal{T}$, is transitive. Then $H / \mathcal{T}$ fixes the vertex $v \in V(\Gamma)$, and so $|H / \mathcal{T}|=t$. Let $\tau \in H$ be of prime order $t$. Then every orbit of $\tau$ has prime order $t$ or has order 1 . If $\tau$ in its action on $T_{v}$ is a $t$-cycle, then $H$ is transitive on $V\left(T_{v}\right)$ and the result follows. Otherwise, $\tau$ is the identity in its action on $V\left(T_{v}\right)$. As $\Gamma$ is connected and $\tau$ has prime order $t \neq 1$, there exists $u \in V(\Gamma)$ such that the action of $\tau$ on $T_{u}$ is the identity, and some vertex $y$ of $T_{u}$ is adjacent to a vertex $z \in V\left(T_{w}\right), w \neq v$, and $z$ is not fixed by $\tau$. Applying $\tau$ to the edge $y z$, we see that $y$ is adjacent to $t$ vertices not in $T_{v}$ and to $t-1$ vertices contained in $T_{v}$. Then the valency of $y$ is $2 t-1$, a contradiction. 
Corollary 3.9. The truncations of neither the Petersen graph nor the Coxeter graph are isomorphic to Cayley graphs.

Proof. As the automorphism groups of the Petersen graph and the Coxeter graph are $S_{5}$ and PGL $(2,7)$ of orders 120 and 336, respectively, according to Theorem 3.8 the truncations of these graphs are Cayley if and only if their automorphism groups contain subgroups of index 4 . Each of these automorphism groups though contain simple groups of index $2\left(A_{5}\right.$ and $\operatorname{PSL}(2,7)$ respectively), and as neither of them are direct products, their only normal proper nontrivial subgroups are $A_{5}$ and $\operatorname{PSL}(2,7)$, respectively. Any subgroup of either of index 4 would then give an embedding into $S_{4}$ by [4, Corollary 4.9], which is solvable.

Theorem 3.10. If $\Gamma$ is a connected vertex-transitive graph of prime valency $p$, then $\mathscr{T}(\Gamma)$ is vertex-transitive if and only if $\operatorname{Aut}(\Gamma)$ contains an element of order $p$ with a fixed point.

Proof. Suppose that $\mathscr{T}(\Gamma)$ is vertex-transitive. Then $\mathcal{T}$ is an $\operatorname{Aut}(\mathscr{T}(\Gamma))$-invariant partition, and so $\left.\operatorname{Stab}_{\operatorname{Aut}(\mathscr{T}(\Gamma))}\left(T_{v}\right)\right|_{T_{v}}$ is transitive on $T_{v}$. As $T_{v}$ has order $p$, it follows that $\left.\operatorname{Stab}_{\text {Aut }(\mathscr{T}(\Gamma))}\left(T_{v}\right)\right|_{T_{v}}$ contains an element of order $p$. Let $\gamma \in \operatorname{Stab}_{\operatorname{Aut}(\mathscr{T}(\Gamma))}\left(T_{v}\right)$ such that $\left.\gamma\right|_{T_{v}}$ has order $p$. Without loss of generality, we assume that $\gamma$ has order $p$ (although we will no longer necessarily have that $\left.\gamma\right|_{T_{v}}$ has order $p$ - for our purposes we only need an element of order $p$ that fixes some $\left.T_{v}\right)$. By Theorem 3.4, Aut $(\mathscr{T}(\Gamma))$ acts faithfully on $\mathcal{T}$, and so $\gamma / \mathcal{T} \neq 1$. Then $\gamma / \mathcal{T}$ has order $p$, fixes the point $v$, and by Theorem 3.4 the permutation $\gamma / \mathcal{T}$ is contained in $\operatorname{Aut}(\Gamma)$.

Suppose that $\operatorname{Aut}(\Gamma)$ contains an element $\gamma$ of order $p$ with a fixed point. As $\Gamma$ is connected, some fixed point $u$ of $\gamma$ is adjacent in $\Gamma$ to some point $u$ that is not fixed by $\gamma$. This follows as there is certainly vertices $x, y \in V(\Gamma)$ with $x$ fixed by $\gamma$ and $y$ not fixed by $\gamma$. We then let $u$ be the first vertex of an $x y$-path in $\Gamma$ that is not fixed by $\gamma$, and $v$ its predecessor on the chosen $x y$-path. As $\gamma$ preserves adjacency, all elements in the (nontrivial) orbit of $u$ are neighbors of $v$. Since $\gamma$ is of prime order $p$, the orbit of $u$ is of length $p$, and since $p$ is the valency of the graph, the orbit of $u$ contains all the neighbors of $v$. Thus, $\operatorname{Aut}(\Gamma)$ acts transitively on the arcs emanating from $v$ and transitively on the vertices of $\Gamma$, and is therefore arc-transitive. The result then follows by Theorem 3.7.

There are a few questions which remain unanswered. First, is it true that Theorem 3.8 holds when the valency $t$ is not prime? Similarly, does Theorem 3.10 hold when the valency is not prime? More specifically, if $\Gamma$ has valency $t$ is it the case that $\mathscr{T}(\Gamma)$ is vertextransitive if and only if $\operatorname{Aut}(\Gamma)$ contains a subgroup of order $t$ with a fixed point (i.e. every element fixes the same point). Finally, what exactly is the group $\operatorname{Stab}_{\operatorname{Aut}(\mathscr{T}(\Gamma))}\left(T_{v}\right)$ in its action on $T_{v}$ ? Of course, as an abstract group it is isomorphic to $\operatorname{Stab}_{\operatorname{Aut}(\Gamma)}(v)$, but what is the action?

Acknowledgement: The authors are indebted to the anonymous referees whose careful reading of this manuscript resulted in suggestions that improved the exposition, results, and proofs in this paper.

\section{References}

[1] N. Biggs, Three remarkable graphs, Canad. J. Math. 25 (1973), 397-411.

[2] G. Exoo and R. Jajcay, Recursive constructions of small regular graphs of given degree and girth, Discrete Math. 312 (2012), 2612-2619, doi:10.1016/j.disc.2011.10.021. 
[3] D. A. Holton and J. Sheehan, The Petersen graph, volume 7 of Australian Mathematical Society Lecture Series, Cambridge University Press, Cambridge, 1993, doi:10.1017/ CBO9780511662058.

[4] T. W. Hungerford, Algebra, volume 73 of Graduate Texts in Mathematics, Springer-Verlag, New York, 1980, reprint of the 1974 original.

[5] K. Kutnar and D. Marušič, Hamilton cycles and paths in vertex-transitive graphs-current directions, Discrete Math. 309 (2009), 5491-5500, doi:10.1016/j.disc.2009.02.017.

[6] A. Malnič, T. Pisanski and A. Žitnik, The clone cover, Ars Math. Contemp. 8 (2015), 95-113.

[7] T. Pisanski and T. W. Tucker, Growth in repeated truncations of maps, Atti Sem. Mat. Fis. Univ. Modena 49 (2001), 167-176, dedicated to the memory of Professor M. Pezzana (Italian).

[8] G. Sabidussi, Vertex-transitive graphs, Monatsh. Math. 68 (1964), 426-438.

[9] W. T. Tutte, A non-Hamiltonian graph, Canad. Math. Bull. 3 (1960), 1-5.

[10] W. T. Tutte, Connectivity in graphs, Mathematical Expositions, No. 15, University of Toronto Press, Toronto, Ont., 1966.

[11] F. Zhang, Y.-C. Chen and Z. Chen, Clique-inserted-graphs and spectral dynamics of cliqueinserting, J. Math. Anal. Appl. 349 (2009), 211-225, doi:10.1016/j.jmaa.2008.08.036. 